\title{
Community history affects the predictability of microbial ecosystem development
}

\author{
Eulyn Pagaling ${ }^{1,2,4}$, Fiona Strathdee ${ }^{1,2}$, Bryan M Spears ${ }^{3}$, Michael E Cates ${ }^{2}$, \\ Rosalind J Allen ${ }^{2,5}$ and Andrew Free ${ }^{1,5}$ \\ ${ }^{1}$ Institute of Cell Biology, School of Biological Sciences, University of Edinburgh, Edinburgh, UK; \\ ${ }^{2}$ SUPA, School of Physics and Astronomy, University of Edinburgh, Edinburgh, UK and ${ }^{3}$ NERC Centre for \\ Ecology and Hydrology Edinburgh, Penicuik, Midlothian, UK
}

\begin{abstract}
Microbial communities mediate crucial biogeochemical, biomedical and biotechnological processes, yet our understanding of their assembly, and our ability to control its outcome, remain poor. Existing evidence presents conflicting views on whether microbial ecosystem assembly is predictable, or inherently unpredictable. We address this issue using a well-controlled laboratory model system, in which source microbial communities colonize a pristine environment to form complex, nutrient-cycling ecosystems. When the source communities colonize a novel environment, final community composition and function (as measured by redox potential) are unpredictable, although a signature of the community's previous history is maintained. However, when the source communities are pre-conditioned to their new habitat, community development is more reproducible. This situation contrasts with some studies of communities of macro-organisms, where strong selection under novel environmental conditions leads to reproducible community structure, whereas communities under weaker selection show more variability. Our results suggest that the microbial rare biosphere may have an important role in the predictability of microbial community development, and that pre-conditioning may help to reduce unpredictability in the design of microbial communities for biotechnological applications.
\end{abstract}

The ISME Journal (2014) 8, 19-30; doi:10.1038/ismej.2013.150; published online 29 August 2013

Subject Category: Microbial population and community ecology

Keywords: community similarity; ecosystem development; microcosms; predictability; rare biosphere

\section{Introduction}

Understanding the development of complex ecological communities is essential for effective biodiversity management and maintenance. It is particularly important to know to what extent community development can be predicted (and hence controlled) and, conversely, to what extent it is inherently unpredictable. This need is especially acute for microbial communities, which are key drivers of the Earth's biogeochemical cycles (Whitman et al., 1998), industrial processes including wastewater treatment, and human gut health (Palmer et al., 2007) and are the subject of ever increasing data sets generated by modern DNA-based community analysis methods

Correspondence: A Free, School of Biological Sciences, University of Edinburgh, Darwin Building, The King's Buildings, Mayfield Road, Edinburgh EH9 3JR, UK.

E-mail: Andrew.Free@ed.ac.uk

${ }^{4}$ Present address: Department of Civil and Environmental Engineering, University of Hawaii at Manoa, Honolulu, HI 96822, USA.

${ }^{5}$ These authors contributed equally to this work.

Received 9 May 2013; revised 23 July 2013; accepted 26 July 2013; published online 29 August 2013
(Prosser et al., 2007; Prosser, 2012). Predictable factors such as environmental selection and interspecies interactions, as well as unpredictable factors such as random dispersal, stochastic population dynamics and priority effects (Chase, 2003, 2007), are all believed to affect microbial community structure and function (Prosser et al., 2007; Langenheder and Székely, 2011). However, the relative importance of these factors remains unclear.

Existing studies on microbial community assembly present conflicting views. Some studies suggest that for a given set of environmental conditions, microbial community development is convergent: common environmental selection recruits the same or similar species from diverse starting species sets to produce similar final community structures (Figure 1; left panel). This view is supported by studies of sulphate-reducing bacteria (SRB) in replicate sediment slurry microcosms (Kurtz et al., 1998), lab-scale activated sludge bioreactors inoculated with wastewater treatment plant communities (Ayarza and Erijman, 2011), soil community transplantation experiments (Lazzaro et al., 2011), microcosm colonization by rainwater bacteria from different sites (Langenheder and 


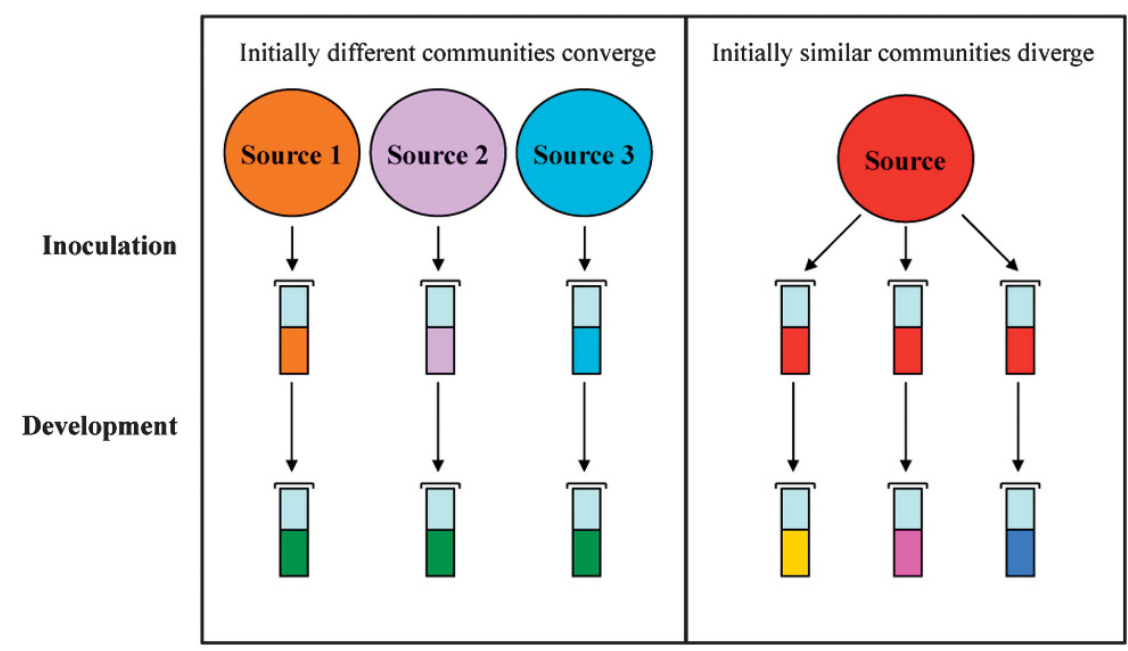

Figure 1 Alternative scenarios for microbial colonization of a novel, pristine environment. Left panel: replicate environments inoculated with different source communities converge toward similar final communities. Right panel: replicate environments inoculated with similar source communities diverge to give distinct final communities.

Székely, 2011) and analysis of the predictability of seasonal changes in microbial community composition in the Pacific Ocean (Fuhrman et al., 2006). In contrast, other studies suggest that microbial community development is divergent: that is, inoculation of identical replicate environments with the same initial microbial community results in different final community structures (Figure 1, right panel). This view is supported by studies of replicate aquatic microcosms inoculated from different environmental sources; after 3 weeks' development, the final communities were no more similar than the starting communities, and inter-replicate variation was high (Langenheder et al., 2006). Similar results have been reported for community development in simple hypolithic communities in desert environments (Caruso et al., 2011), wastewater treatment plants (Ofiteru et al., 2010), replicate laboratory phototrophic biofilms (Roeselers et al., 2006) and laboratory-scale wetlands (Baptista et al., 2008). It is important to note, however, that variable final community composition may be associated with stable broad-scale ecosystem function (Fernández et al., 1999; Langenheder et al., 2006).

For macro-organisms, there is some evidence that the relative importance of predictable and unpredictable factors in community development depends on the degree of environmental harshness (Chase, 2007) or disturbance (Violle et al., 2010). Pioneering work on the effect of drought on the assembly of macro-organisms in pond ecosystems (Chase, 2007) demonstrated that increasing the strength of environmental selection increases the reproducibility of the final community structure; this conclusion is supported by other studies of a variety of natural environments (Donohue et al., 2009; Lepori and Malmqvist, 2009; Helmus et al., 2010; Myers and Harms, 2011), as well as by microcosm studies of eukaryotic microbial (protist) community assembly (Weatherby et al., 1998; Jiang and Patel, 2008). However, it is unclear whether these principles also apply to complex natural microbial communities. Microbial communities show much greater metabolic diversity than those of macro-organisms, and also typically possess a highly-diverse 'rare biosphere' of species that are present at very low abundance (Sogin et al., 2006; Pedrós-Alió, 2012). Microorganisms also display shorter generation times, more rapid adaptive evolution and greater dispersal than macroorganisms. These factors may lead to a different disturbance-predictability relationship for microbial communities, compared with that of macro-organisms. Whether such factors can account for the apparently conflicting results on microbial community divergence or convergence is at present unclear. Interestingly, in simple microbial systems, evolutionary history has also been shown to influence the diversification of individual species during community assembly (Knope et al., 2012).

In this study, we consider whether the previous history of a complex microbial community affects the variability of its response to environmental selection. We address this issue using wellcontrolled laboratory experiments with replicated freshwater sediment-water microcosms. Microcosms provide a powerful tool for microbial ecology (Jessup et al., 2004), as they make it possible to perform replicate laboratory experiments under manipulable conditions. Many important advances have been made using 'simple' microcosm communities with relatively few species (Weatherby et al., 1998; Langenheder and Székely, 2011; Hekstra and Leibler, 2012). In contrast, in this study we aim to preserve the key features of natural microbial ecosystems-high microbial diversity, nutrient cycling, community-environment feedbacks, strong interspecies interactions and spatial structure. Our system of freshwater microcosms, based on 
the Winogradsky column (Madigan et al., 2011), retains these properties while allowing us to manipulate the extent of environmental selection or disturbance, to compare initial and final community composition and to measure community function in the form of a redox potential gradient.

In our experiments, groups of freshwater microorganisms colonize pristine (sterile) microcosm environments to form complex nutrient-cycling communities. By comparing final community composition and function among microcosms formed from the same and different source communities, we are able to test for convergence of initially different communities (Figure 1, left panel) or divergence of initially similar communities (Figure 1, right panel). Our results show that when colonization involves a drastic change in habitat (large environmental disturbance), the final community composition and function are unpredictable, with a high degree of variation between replicate experiments but retaining a signature of the source community. In contrast, when the community colonizes an environment to which it is already pre-conditioned, the final community composition can be more predictable.

\section{Materials and methods}

\section{Sampling}

Sediment and water samples were collected from six freshwater lochs and a freshwater pond in Scotland in September 2008 and October 2008 (Supplementary Table S1). These sources cover a range of sizes (surface area $0.01-14 \mathrm{~km}^{2}$ ), elevations $(30-300 \mathrm{~m})$, mean water $\mathrm{pH}$ values (6.16-8.70) and nutrient levels (Supplementary Table S1). Sediment cores were taken from depths of $0.8-2.0 \mathrm{~m}$ using a hand-held corer, except at Loch Leven where a Jenkin's sediment sampler was used to sample from a depth of $3.8 \mathrm{~m}$. Water and sediment sub-samples were then taken from the cores using sterile $50 \mathrm{ml}$ Falcon tubes. Microbial diversity in these samples was characterized by denaturing gradient gel electrophoresis (DGGE) fingerprinting of the V3 regions of 16S rRNA genes from Bacteria and Archaea (Fernández et al., 1999).

\footnotetext{
Microcosms

Our pond sediment-water microcosms are based on the well-known Winogradsky column (Madigan et al., 2011). This system, originally designed to select for anaerobes, consists of a lower layer of pond sediment, mixed with nutrients (cellulose and sulphate) and an upper layer of water. Upon illumination for several weeks, the microcosm develops into a stratified, nutrient-cycling ecosystem. To create pristine microcosm environments, pond sediment and water from Blackford Pond in Edinburgh were sieved ( $1 \mathrm{~mm}$ pore size) to remove debris, and nutrients added: $0.25 \mathrm{~g}$ of $\mathrm{CaCO}_{3}$ (buffer
}

and $\mathrm{CO}_{2}$ source), $2.5 \mathrm{~g}$ of cellulose (carbon source) and $5 \mathrm{~g}$ of $\mathrm{CaSO}_{4}$ (sulphur source) per $100 \mathrm{~g}$ of sediment. The mixture was thoroughly homogenized and aliquoted into $15 \mathrm{ml}$ Falcon tubes $(7.5 \mathrm{~g}+5 \mathrm{ml}$ pond water per tube). The microcosms were then sterilized by autoclaving at $121^{\circ} \mathrm{C}$ for $15 \mathrm{~min}$ and stored at $4{ }^{\circ} \mathrm{C}$. Triplicate sterilized microcosms were inoculated with $100 \mathrm{mg}$ sediment and $100 \mu \mathrm{l}$ water from the seven environmental sites in a microbiological cabinet, before being thoroughly homogenized, then incubated under constant Northlight illumination in an incubator for 16 weeks at $25^{\circ} \mathrm{C}$, after which visible changes in the microcosms (for example, colour changes) had largely ceased; additional tests demonstrate that redox potential and bacterial community structure also stabilize in this microcosm system by 16 weeks (Supplementary Figure S1). Sterilized microcosms without inoculation did not develop redox potential gradients or colour changes when incubated under the same conditions for 19 weeks. For inoculation of 'daughter' microcosms with pre-conditioned microcosm communities, two of eight mature (16 week) microcosms, originally set up using Blackford Pond sediment and water, were homogenized and $100 \mu \mathrm{l}$ of the slurry was used to inoculate eight replicate sterilized microcosms, which were incubated as above.

\section{Community DNA extraction and PCR amplification}

Sediments from environmental samples and developed microcosms (sediment and water) were homogenized by vortexing. Community DNA was then extracted from $1 \mathrm{~g}$ sediment, $1 \mathrm{ml}$ water or $1 \mathrm{ml}$ homogenized microcosm using an UltraClean Soil DNA Isolation Kit (MoBio, Carlsbad, CA, USA). Variable (V3) regions of bacterial and archaeal 16S rRNA genes were amplified for DGGE via a nested PCR approach using the primers and conditions listed in Table 1; $d s r B$ gene fragments were amplified similarly but via a single round of PCR (Table 1). For 454 sequencing of bacterial 16S rRNA gene V3 regions from initial Loch Leven sediment, nested primers containing 454 adapter sequences (Table 1; Supplementary Methods) were used. All PCR reactions were set up in a PCR6 Vertical Laminar Airflow Cabinet with UV sterilization (Labcaire Systems, Bristol, UK); both reaction tubes and PCR mixtures were treated for 15 min with $15 \mathrm{~W}$ UV light (wavelength $=254 \mathrm{~nm}$ ) to destroy contaminating DNA, prior to the addition of dNTPs, Taq polymerase and template DNA (Padua et al., 1999). Negative controls containing no added DNA template were routinely amplified alongside both rounds of functional PCR reactions.

Denaturing gradient gel electrophoresis

Nested PCR products were separated on DGGE gels (McCaig et al., 2001) containing a linear gradient 
Table 1 Summary of primers and PCR conditions used

\begin{tabular}{|c|c|c|c|c|c|}
\hline Target & Primer & Positions $^{\mathrm{a}}$ & $\begin{array}{l}\text { PCR } \\
\text { round }\end{array}$ & Thermal cycling conditions & Reference \\
\hline Bacteria (16S rRNA genes), DGGE & $\begin{array}{l}\text { BactF } \\
\text { BactR }\end{array}$ & $\begin{array}{l}8-27 \\
1541-1522\end{array}$ & $\begin{array}{l}1 \\
1\end{array}$ & $\begin{array}{l}94^{\circ} \mathrm{C} \text { for } 5 \mathrm{~min} \text {; followed by } 30 \text { cycles of } 94^{\circ} \mathrm{C} \text { for } 1 \mathrm{~min} \text {, } \\
55^{\circ} \mathrm{C} \text { for } 1 \mathrm{~min}, 72^{\circ} \mathrm{C} \text { for } 3 \mathrm{~min} \text {; followed by } 72^{\circ} \mathrm{C} \text { for } 7 \mathrm{~min} .\end{array}$ & Fernández et al., 1999 \\
\hline & $\begin{array}{l}341 \mathrm{~F}-\mathrm{GC} \\
534 \mathrm{R}\end{array}$ & $\begin{array}{l}341-357 \\
534-518\end{array}$ & $\begin{array}{l}2 \\
2\end{array}$ & $\begin{array}{l}95^{\circ} \mathrm{C} \text { for } 5 \mathrm{~min} \text {; followed by } 28 \text { cycles of } 94^{\circ} \mathrm{C} \text { for } 1 \mathrm{~min} \text {, } \\
64^{\circ} \mathrm{C} \text { for } 1 \mathrm{~min}, 72^{\circ} \mathrm{C} \text { for } 1 \mathrm{~min} 30 \mathrm{~s} \text {; followed by } 72^{\circ} \mathrm{C} \text { for } 10 \mathrm{~min} \text {. }\end{array}$ & Muyzer et al., 1993 \\
\hline Archaea (16S rRNA genes), DGGE & $\begin{array}{l}\text { ArchF } \\
\text { ArchR } \\
\text { rSAf(i) } \\
\text { PARCH519r }\end{array}$ & $\begin{array}{l}55-64 \\
913-895 \\
316-332 \\
465-451\end{array}$ & $\begin{array}{l}1 \\
1 \\
2 \\
2\end{array}$ & $\begin{array}{l}94^{\circ} \mathrm{C} \text { for } 2 \mathrm{~min} \text {; followed by } 35 \text { cycles of } 94^{\circ} \mathrm{C} \text { for } 30 \mathrm{~s} \text {, } \\
52^{\circ} \mathrm{C} \text { for } 1 \mathrm{~min}, 72^{\circ} \mathrm{C} \text { for } 3 \mathrm{~min} \text {; followed by } 72^{\circ} \mathrm{C} \text { for } 7 \mathrm{~min} \text {. } \\
95{ }^{\circ} \mathrm{C} \text { for } 5 \mathrm{~min} \text {; followed by } 5 \text { cycles of } 94^{\circ} \mathrm{C} \text { for } 30 \mathrm{~s}, 58^{\circ} \mathrm{C} \text { for } 30 \mathrm{~s} \text {, } \\
72^{\circ} \mathrm{C} \text { for } 1 \mathrm{~min} \text {; followed by } 30 \text { cycles of } 92^{\circ} \mathrm{C} \text { for } 30 \mathrm{~s}, 63^{\circ} \mathrm{C} \text { for } \\
30 \mathrm{~s}, 72^{\circ} \mathrm{C} \text { for } 1 \text { min; followed by } 72{ }^{\circ} \mathrm{C} \text { for } 10 \mathrm{~min} .\end{array}$ & $\begin{array}{l}\text { Fernández et al., } 1999 \\
\text { Nicol et al., } 2005\end{array}$ \\
\hline Sulphate reducers $(d s r B)$, DGGE & $\begin{array}{l}\text { DSRp2060F-GC } \\
\text { DSR4R }\end{array}$ & $\begin{array}{l}420-440 \\
797-781\end{array}$ & $\begin{array}{l}1 \\
1\end{array}$ & $\begin{array}{l}94^{\circ} \mathrm{C} \text { for } 4 \mathrm{~min} \text {; followed by } 35 \text { cycles of } 94^{\circ} \mathrm{C} \text { for } 1 \mathrm{~min}, 55^{\circ} \mathrm{C} \\
\text { for } 1 \mathrm{~min}, 72^{\circ} \mathrm{C} \text { for } 1 \mathrm{~min} \text {; followed by } 72^{\circ} \mathrm{C} \text { for } 10 \mathrm{~min} \text {. }\end{array}$ & Geets et al., 2006 \\
\hline $\begin{array}{l}\text { Bacteria (16S rRNA genes), } \\
454 \text { sequencing }\end{array}$ & $\begin{array}{l}341 \mathrm{~F}-454 \mathrm{~F} \\
534 \mathrm{R}-454 \mathrm{R}\end{array}$ & $\begin{array}{l}341-357 \\
534-518\end{array}$ & $\begin{array}{l}2 \\
2\end{array}$ & $\begin{array}{l}95^{\circ} \mathrm{C} \text { for } 5 \mathrm{~min} \text {; followed by } 28 \text { cycles of } 94^{\circ} \mathrm{C} \text { for } 1 \mathrm{~min}, 64^{\circ} \mathrm{C} \\
\text { for } 1 \mathrm{~min}, 72^{\circ} \mathrm{C} \text { for } 1 \mathrm{~min} 30 \mathrm{~s} \text {; followed by } 72^{\circ} \mathrm{C} \text { for } 10 \mathrm{~min} .\end{array}$ & This study \\
\hline
\end{tabular}

Components of the PCR reactions are listed in Supplementary Methods.

aPositions correspond to Escherichia coli numbering for bacterial 16S rRNA genes, Methanococcus maripaludis numbering for archaeal 16S rRNA genes and Desulfovibrio vulgaris numbering for bacterial $d s r B$ genes.

of $30-70 \%$ denaturant (where $100 \%$ denaturant is defined as $7 \mathrm{M}$ urea $(42 \% \mathrm{w} / \mathrm{v})$ and $40 \%(\mathrm{w} / \mathrm{v})$ formamide). For DGGE analysis of $d s r B$ amplicons, a denaturant gradient of $40-80 \%$ was used. Electrophoresis was carried out on a DCode Universal Mutation Detection System (Bio-Rad, Hemel Hempstead, UK) in $7 \mathrm{~L}$ of $1 \times \mathrm{TAE}$ buffer at a constant temperature of $60^{\circ} \mathrm{C}$ for $960 \mathrm{~min}$ at $75 \mathrm{~V}$. Silver staining of gels was carried out as previously described (Nicol et al., 2005). All DGGE gels included three lanes with a standard set of 11 marker bands (see Supplementary Methods). DGGE fingerprints obtained from replicate PCRs of the same DNA extraction or replicate DNA extractions from the same microcosm were essentially identical (Supplementary Figure S1).

\section{Redox potentials}

Redox potential gradients were measured using a heated platinum wire, which was pierced into the side of the developed microcosms at three positions: in the upper part of the water layer, at the bottom of the sediment layer and $5 \mathrm{~mm}$ below the watersediment interface. The potential of the wire was measured relative to an $\mathrm{Ag} / \mathrm{AgCl}$ reference electrode (Radiometer Analytical, Villeurbanne, France), which was inserted into the top of the microcosm water layer, using a WG020 voltmeter (Precision Gold) set at a sensitivity of $2 \mathrm{~V}$.

\section{DGGE fingerprint analysis}

BioNumerics version 6.0 (Applied Maths, SintMartens-Latem, Belgium) was used to produce normalized composite gels from the DGGE gels, using the marker lanes as a reference. Band-matching data with band intensities were imported into Primer 6 Version 6.1.12 (Primer-E, Ivybridge, UK) and used to construct Bray-Curtis similarity matrices following square root transformation to avoid domination by the most abundant species. The Bray-Curtis coefficient accurately captures diversity differences in various types of model data sets (Kuczynski et al., 2010). An index of 100 indicates identical fingerprints, whereas an index of 0 indicates no common bands (Clarke and Warwick, 2001). Nonparametric multi-dimensional scaling (NMDS) plots generated from the BrayCurtis similarity matrices in Primer 6 were used to represent the distance between each sample in twodimensional space (Clarke and Warwick, 2001). NMDS was performed using 100 random starting configurations of sample points; the accuracy of the NMDS representation was determined by calculating the Kruskal stress (Kruskal, 1964). Primer 6 was also used for additional statistical analysis of the DGGE fingerprints and other parameters. Analysis of similarities (ANOSIM) and analysis of variance (ANOVA) (Sokal and Rohlf, 1969; Clarke and Warwick, 2001) were used to assess the significance of the source lochs in determining the observed similarity matrix and the mean microcosm redox potential, respectively. PERMANOVA and PERMDISP analyses (Anderson et al., 2008) were carried out using the PERMANOVA + add-on to Primer 6 to test for significant differences in the distribution and dispersion, respectively, of sets of communities based on Bray-Curtis similarities. For comparisons focusing only on within-set variability, sets of replicate samples inoculated with different source communities were mapped onto a common centroid in multivariate space prior to carrying out PERMDISP analysis, in order to separate inter-replicate variation from variation between replicate sets (see Supplementary Methods). Similarity percentage (SIMPER) analysis (Clarke and Warwick, 2001) was used to investigate the contributions of individual bands (species) to the Bray-Curtis dissimilarity (100 - similarity) between the archaeal 16S rRNA gene fingerprints obtained from the microcosms. 


\section{Results and discussion}

\section{Source communities}

Source microbial communities comprised sediment and water samples from six freshwater lochs and a freshwater pond in Scotland (Supplementary Table S1). The bacterial and archaeal subcommunities in these samples were characterized by DGGE fingerprinting of the V3 region of the respective 16S rRNA genes (Supplementary Figure S2; see Materials and Methods). For the bacterial communities, the mean number of DGGE bands obtained from the water samples was $25.1 \pm 1.7$ (all error ranges quoted are \pm s.e.m.) (Supplementary Table S2; see also the $\mathrm{Rr}$ and $e^{\mathrm{H}^{\prime}}$ diversity measures in this table), while the sediment samples were so taxon rich that the resulting fingerprints could not be resolved. For the archaeal communities, the mean number of bands for the sediment samples was $26.7 \pm 1.5$ (Supplementary Table S2); however, archaeal V3 regions could not be amplified from the water samples, suggesting very low abundance of Archaea in the water. For the bacterial water communities and the archaeal sediment communities, the mean Bray-Curtis similarities were $41.6 \pm 2.1$ and $44.7 \pm 1.8$, respectively (Supplementary Table S2). Therefore, the source samples clearly differed in both bacterial and archaeal community composition. Similar biogeographical variation (Martiny et al., 2006) was seen for DGGE fingerprints of the bacterial dissimilatory sulphite reductase $(d s r B)$ gene, specific to SRB (Geets et al., 2006), in our samples (Supplementary Table S2).

\section{Colonization of a common, unfamiliar microcosm environment}

Our seven different source communities were allowed to colonize identical, pristine microcosm environments, under temperature and nutrient conditions very different from those in their source lochs, thus imposing strong environmental selection. Mixed water and sediment from each source were used to inoculate identical triplicate microcosms. During incubation, a vertical redox potential gradient develops in the microcosms as a result of microbial activity, with less negative redox potentials in the upper layers of the microcosms (Supplementary Table S3), indicating differences in the availability of electron acceptors at different levels of the stratified system. Both redox potential gradient and microbial community structure stabilize after 16 weeks' incubation (Supplementary Figure S1). However, the final redox potential gradient varied considerably among the microcosms (Figure 2), suggesting that different functional (redox) states can result from the community development process. There were significant differences in the vertically averaged redox potential dependent on the source community (ANOVA,
$\left.F_{6,14}=4.435, \quad P=0.01\right)$; however, as evident in Figure 2, there was also a large degree of functional unpredictability between replicate microcosms derived from the same inoculum. Consistent with this, the estimated contributions to the variability in redox potential due to source loch and interreplicate variation were $43 \%$ and $57 \%$, respectively (Clarke and Warwick, 2001).

Unpredictable, but source-dependent, community composition following colonization of an unfamiliar environment

We next analysed the variability of the final community composition in our microcosms. As one might expect given the small size of the microcosms and the strong selection, community richness was substantially reduced compared with the inoculating sediments: mean band numbers were $22.8 \pm 1.1$ for bacterial $16 \mathrm{~S}$ rRNA genes and $14.0 \pm 0.7$ for archaeal 16S rRNA genes (Supplementary Figure S2), with similar decreases for $\mathrm{Rr}$
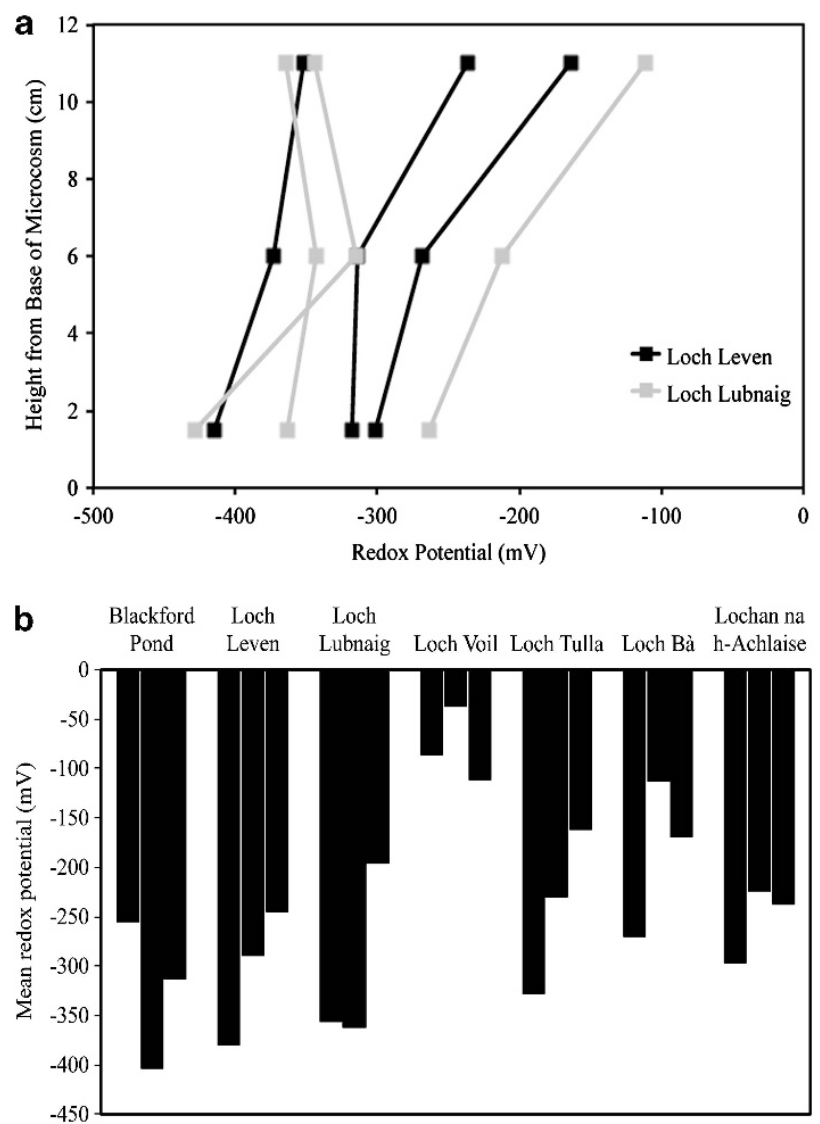

Figure 2 Community function within the developed microcosms differs between replicates. (a) Redox potential measurements taken in the developed (16 weeks) Loch Leven and Loch Lubnaig microcosms near the base of the sediment $(2 \mathrm{~cm})$, just below the sediment-water interface $(6 \mathrm{~cm})$ and near the top of the water layer $(11 \mathrm{~cm})$. The full set of data is presented in Supplementary Table S3. (b) Microcosm redox potentials (means of the measurements at the three different heights in (a)) are shown for each of the three replicate microcosms from each source after 16 weeks' development. 
and $e^{\mathrm{H}^{\prime}}$ (Supplementary Table S2). The lower $e^{\mathrm{H}^{\prime}}$ values also reflect a less even community composition in the microcosms compared with the inocula. SRB also showed reduced diversity and evenness in the microcosm communities as measured by band number, $\mathrm{Rr}$ and $e^{\mathrm{H}^{\prime}}$ (Supplementary Table S2).

DGGE analysis showed significant differences in composition between the microcosm communities after development in the common environment. Three aspects of the variation in community composition among our microcosms were notable. First, triplicate microcosms inoculated from the same environmental source showed low similarity (Figure 3): mean inter-replicate Bray-Curtis similarities were $57.3 \pm 1.9$ for Bacteria and $47.7 \pm 4.1$ for Archaea (Supplementary Table S2), suggesting substantial unpredictability in the community development. Second, despite this inter-replicate variability, the source community also influenced microcosm community structure: mean similarities between microcosms with different inocula were substantially lower (37.2 \pm 0.5 for Bacteria and $27.4 \pm 0.7$ for Archaea; Supplementary Table S2) than between those with the same inoculum. One-way ANOSIM tests (Clarke and Warwick, 2001) confirmed that the microcosm communities clustered according to source loch: $R=0.914, P<0.001$ for Bacteria; $R=0.646, P<0.001$ for Archaea; $R=0.651, P<0.001$ for SRB. PERMANOVA analysis also showed a significant effect of source loch on final microcosm community structure for all three genes (pseudo- $F$ statistics between 2.98 and $4.47 ; P=0.001$; Supplementary Table S2). Indeed, our PERMANOVA analysis showed that, for both Bacteria and Archaea, variability between replicate microcosm communities and effects of source loch made approximately equal contributions to the total variation among microcosm communities, whereas for SRB, the variability between replicates was more important than the effect of source loch (Supplementary Table S2).

A third interesting aspect of our results was that microcosm communities diverged in composition compared with the source communities. For Archaea, mean microcosm similarity was $30.3 \pm 0.7$ compared with $44.7 \pm 1.8$ for the initial sediment samples (Figure 3; Supplementary Table S2), whereas for SRB, the corresponding microcosm and sediment similarities were $18.9 \pm 0.6$ and $30.6 \pm 1.9$, respectively (Supplementary Figure S3; Supplementary Table S2). PERMDISP analysis (Clarke and Warwick, 2001) was used to test whether the dispersion between all microcosms was greater than that between inocula: this was true for both Archaea $(F=22.34 ; P=0.002)$ and SRB $(F=12.91 ; \quad P=0.007 ; \quad$ Supplementary Table S2). Thus different source communities diverged, rather than converged, upon colonization of the common microcosm environment. For Bacteria, where only water samples, which do not contain most of
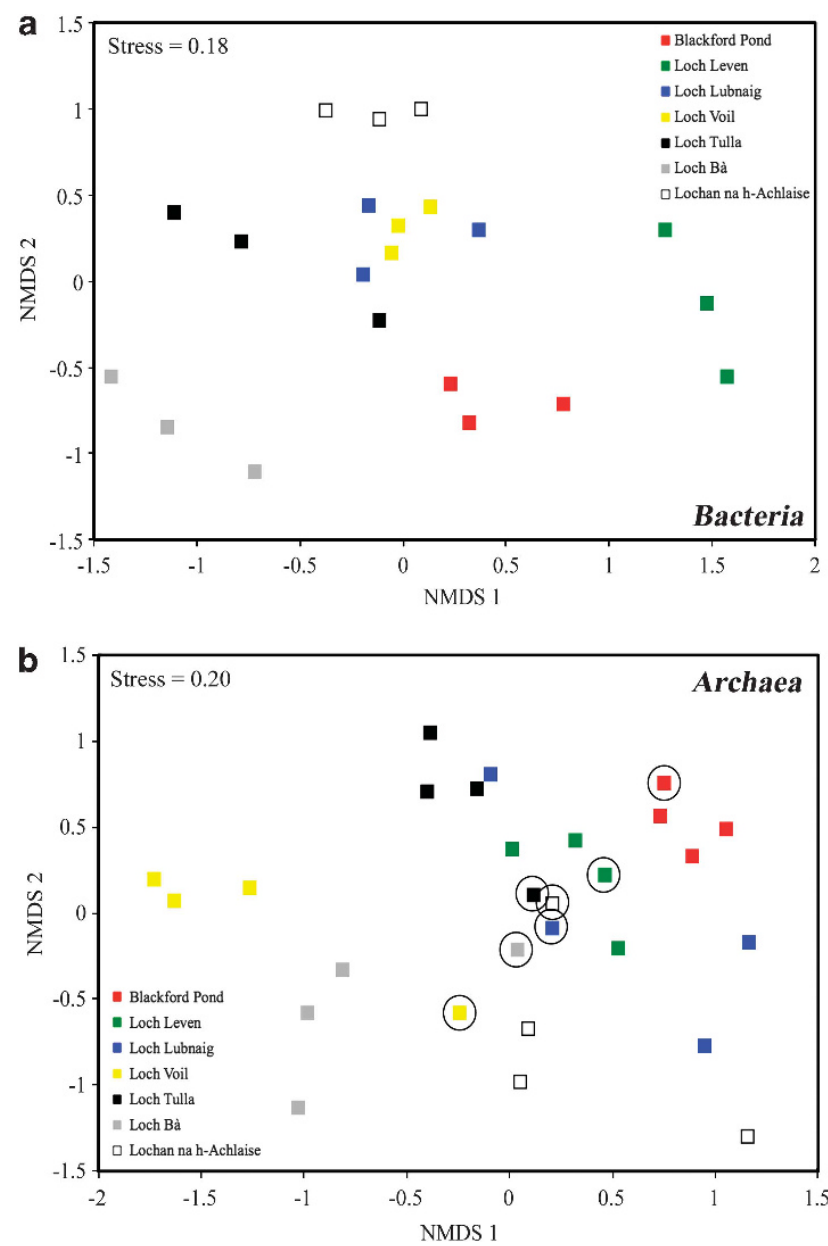

Figure 3 Differences in community structure between the developed microcosms. NMDS plots of Bray-Curtis similarity between the DGGE fingerprints of bacterial (a) and archaeal (b) 16S rRNA genes from triplicate developed microcosms after 16 weeks' development show that triplicate microcosms differ in their final community compositions, whereas different source communities produce divergent microcosm communities. The corresponding fingerprints are shown in Supplementary Figure S2b. For the archaeal communities the plot also shows the sediment inocula (circled); equivalent results for bacterial $d s r B$ genes are shown in Supplementary Figure S3.

the inoculum bacterial diversity, gave resolvable fingerprints, we could not perform this analysis.

\section{Amplification of rare species upon community} rearrangement in an unfamiliar environment

Differences in community composition between replicate microcosms could result from the random presence or absence of rare species in the inocula, if initially rare species are amplified as the unfamiliar environment is colonized. To investigate this hypothesis, we compared the abundance of individual archaeal taxa in the source sediments and the developed microcosms. Indeed, many taxa that were undetectable in the source were abundant in the microcosms, and vice versa (Figure 4a) (defining 'undetectable' as below the DGGE sensitivity threshold, which is estimated to be $1 \%$ of the total 

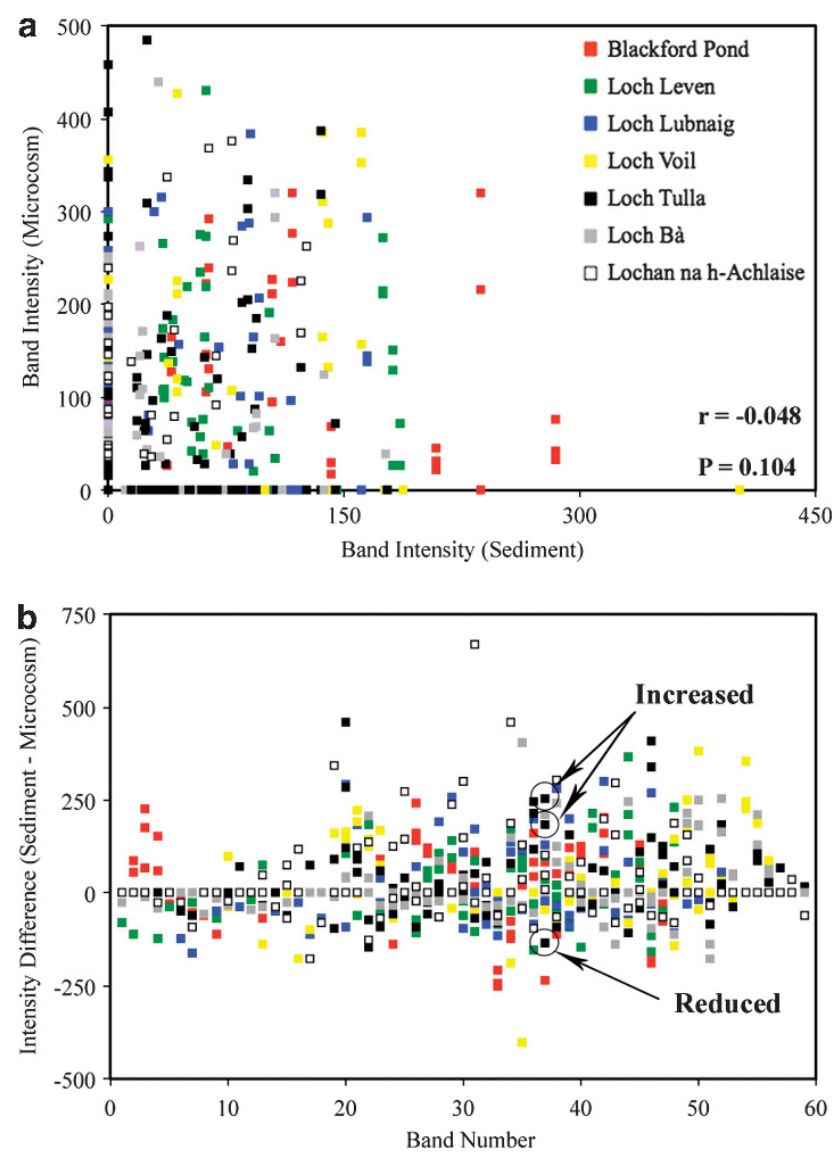

Figure 4 Archaeal taxon abundance in the source sediment does not correlate with that in the developed microcosms. Analysis of intensity of individual bands within the archaeal 16S rRNA gene DGGE fingerprints (a) shows no significant correlation between intensity in the sediment sample and intensity in each of the triplicate microcosms. (b) Selective effects on individual taxa differ between replicate microcosms. The difference in band intensity between the source sediment sample and each of the triplicate microcosms for each of the individual bands in (a) is shown. An example in which a single taxon is increased and decreased in abundance in different microcosm replicates (from the Loch Tulla source) is indicated. Colour codes are as in (a).

community (Muyzer et al., 1993)), suggesting that initially rare taxa are selected in our experiments. Even for those Archaea which were detectable in both the source and microcosm communities, we found no significant correlation between initial and final abundance (Pearson $R=-0.048 ; P=0.104$ ). For the bacterial community, where inoculating sediment fingerprints could not be resolved, we employed instead a high-throughput sequencing approach to study the initial sediment community. We obtained 10277 pyrosequences of 16S rRNA gene V3 regions from the Loch Leven sediment sample (Supplementary Methods), and searched them for 28 sequences of cloned bacterial 16S rRNA gene V3 regions derived from the corresponding developed microcosms. Only 6 of these 28 sequences, which correspond to species which were abundant in the microcosms, were detectable $(\geqslant 98 \%$ sequence identity) in the pyrosequencing data set (Supplementary Table S4). These results show that drastic community rearrangement occurred upon transfer to the unfamiliar microcosm environment, probably driven by strong selection for taxa different from those which were abundant in the source-many of which were rare in the source communities.

Interestingly, our analysis also showed that the selective effects on a given taxon during community development differed between replicate microcosms (Figure 4b)-individual archaeal taxa were amplified in some replicates but suppressed in others. For example, band no. 37 was amplified in two replicates of the Loch Tulla-derived microcosms compared with the inoculating sediment, but reduced in intensity in the third replicate (Figure 4b). It is also clear that many taxa were amplified in microcosms derived from some sources, but suppressed in microcosms from other sources. A similar effect was seen for individual SRB taxa (Supplementary Figure S4). This suggests that there is no unifying environmental selection across the group of microcosms; rather, the complex dynamics caused by community-environment feedbacks and interspecies interactions, and/or stochastic effects, create different selective environments in replicate microcosms. In our microcosms, metabolic feedbacks may lead to non-linear ecosystem dynamics, potentially producing chaotic or initial condition-dependent trajectories as observed in other studies (Becks et al., 2005; Graham et al., 2007; Benincà et al., 2008). Indirect interspecies interactions mediated by the physical environment are also likely to have a key role in our microcosms: for example, the generation of an anaerobic state as a result of cellulose degradation and sulphate reduction, which will strengthen selection for anaerobes in the developing community.

Does amplification of the rare biosphere cause unpredictability in community development? To test for possible effects of rare species (undetectable by DGGE) on community divergence, we asked whether taxa that are rare in the source communities are also more variable in the final communities. Quantification of the contributions of individual archaeal taxa to the Bray-Curtis dissimilarity within triplicate groups of microcosms using SIMPER (see Materials and methods) did not suggest increased contributions to microcosm dissimilarity for taxa that were undetectable in the source sediment (that is, with zero DGGE intensity) compared with those that were detectable in the source (Supplementary Figure S5). This does not, however, rule out the hypothesis that rare species are responsible for unpredictable community development. While the naïve view in which individual species make independent contributions to community variability clearly does not hold for our system, if interspecies interactions are strong then the random presence/ 


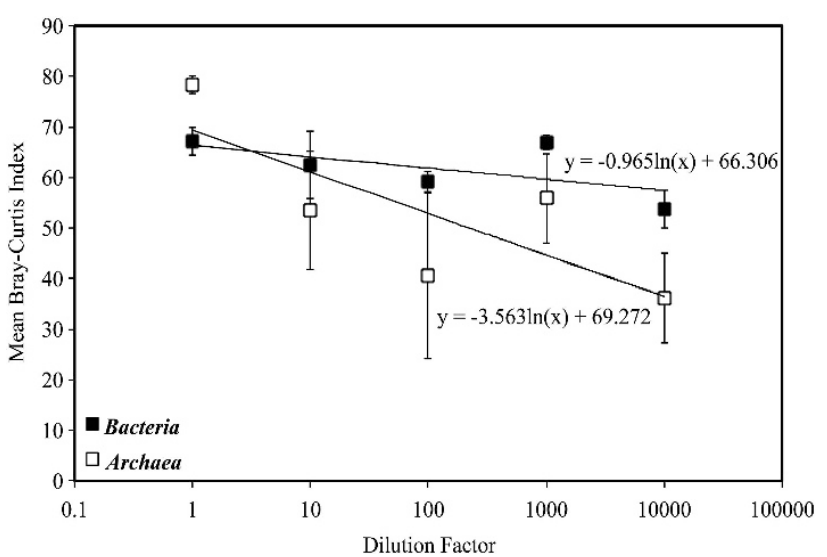

Figure 5 Effect of dilution of the inoculum on community similarity. A plot of Bray-Curtis similarity index (mean \pm s.e.m.) of the bacterial and archaeal 16S rRNA gene fingerprints from the triplicate microcosms versus dilution factor of sediment + water inoculum. Dilution factor $=1$ corresponds to the standard inoculum size used in other experiments. Best-fit trend lines and their slopes are indicated; the effect of inoculum dilution on similarity is significant for Archaea, but not for Bacteria.

absence of rare archaeal species in the inocula may lead to variability in the final abundance not only of those species but also of others, which were not rare in the inocula. The same analysis could not be performed for the bacterial community due to the lack of resolvable 16S rRNA gene fingerprints from the inoculating sediments.

As another test for a causative role of the rare biosphere in unpredictable community development, we systematically decreased the size of the source community, by inoculating triplicate microcosms with serially-diluted Blackford Pond sediment and water samples (see Supplementary Methods). Microcosms seeded from smaller inocula should be more subject to the random presence or absence of rare species and therefore, according to our hypothesis, their final composition should be more variable (Langenheder et al., 2006; Wertz et al., 2007). Consistent with this hypothesis, the mean inter-replicate Bray-Curtis similarity index for Archaea in the developed microcosms decreased significantly from $\sim 80$ to $\sim 40$ over four orders of magnitude of dilution of the inoculum $(R=-0.55$; $P=0.034$; Figure 5). For Bacteria, however, only a slight decrease in similarity, which was not statistically significant $(R=-0.43 ; \quad P=0.113)$ was observed. Thus, while our data show that rare species are amplified and may have a role in causing unpredictability, the full story may be more complex. Ongoing high-throughput sequence analysis of the initial inocula and developed microcosm communities should assist in assessing this role.

\section{Colonization of a familiar environment is more predictable}

In our experiments so far, the source microbial communities experienced very different environmental conditions in the microcosms compared with those in their source lochs, leading to extensive community rearrangement and, probably, selection for initially rare species. We hypothesized that the results might be different if microcosms were colonized by communities which were already 'conditioned' to the microcosm environment of constant illumination, mesophilic temperature, high organic matter content and high sulphate-that is, communities, which do not undergo extensive rearrangement upon colonizing the microcosm. This can be achieved by using, as inocula, samples from homogenized mature microcosms.

To test this hypothesis, we set up eight replicate microcosms A-H (see Methods) derived from Blackford Pond sediment (we used eight replicates to provide better statistics). The developed microcosms showed significant variation in bacterial community composition (mean Bray-Curtis index $=34.2 \pm 2.2$; Supplementary Table S2). Two of these pre-conditioned communities (A and $\mathrm{H}$ ) were each then used to inoculate a further eight replicate microcosms, and the developed bacterial community compositions analysed. As expected, community rearrangement was less extensive for the communities pre-conditioned to the microcosm environment than for those taken directly from Blackford Pond (Figure 6). For the pre-conditioned source communities, we observed significant correlation between the abundance of bacterial taxa in the source community and their abundance in the developed microcosm communities $(R=0.452$, $P<0.0001$ for source A; $R=0.222, P=0.002$ for source H; Figure 6b), in contrast to microcosms inoculated with environmental samples, where no correlation was observed (Figure 4).

To test whether community development was indeed more predictable when the microcosms were colonized by pre-conditioned communities, we calculated the average Bray-Curtis similarity between replicate microcosms seeded from the same pre-conditioned inoculum. This was $70.0 \pm 1.2$, substantially higher than in the original eight microcosms (34.2 \pm 2.2 ; Supplementary Table S2), a highly significant difference as determined by PERMDISP $(F=89.03 ; P=0.001)$. (We also compared the dispersion among replicate microcosms seeded from 'pre-conditioned' Blackford Pond microcosm communities with that among replicate microcosms seeded with environmental samples from all the sources sampled. Here we found, in agreement with our hypothesis, that the mean BrayCurtis similarity was greater for the former than the latter $(57.3 \pm 1.9$ versus $70.0 \pm 1.2$; Supplementary Table S2). However, the PERMDISP comparison showed only marginal significance $(F=3.56$; $P=0.069)$.). The NMDS plot (Figure 6a) also shows that groups of replicate microcosms seeded with the same pre-conditioned source community tend to cluster together with their source community, as demonstrated by a between-group mean Bray-Curtis similarity of $42.8 \pm 0.8$ (Supplementary Table S2). 

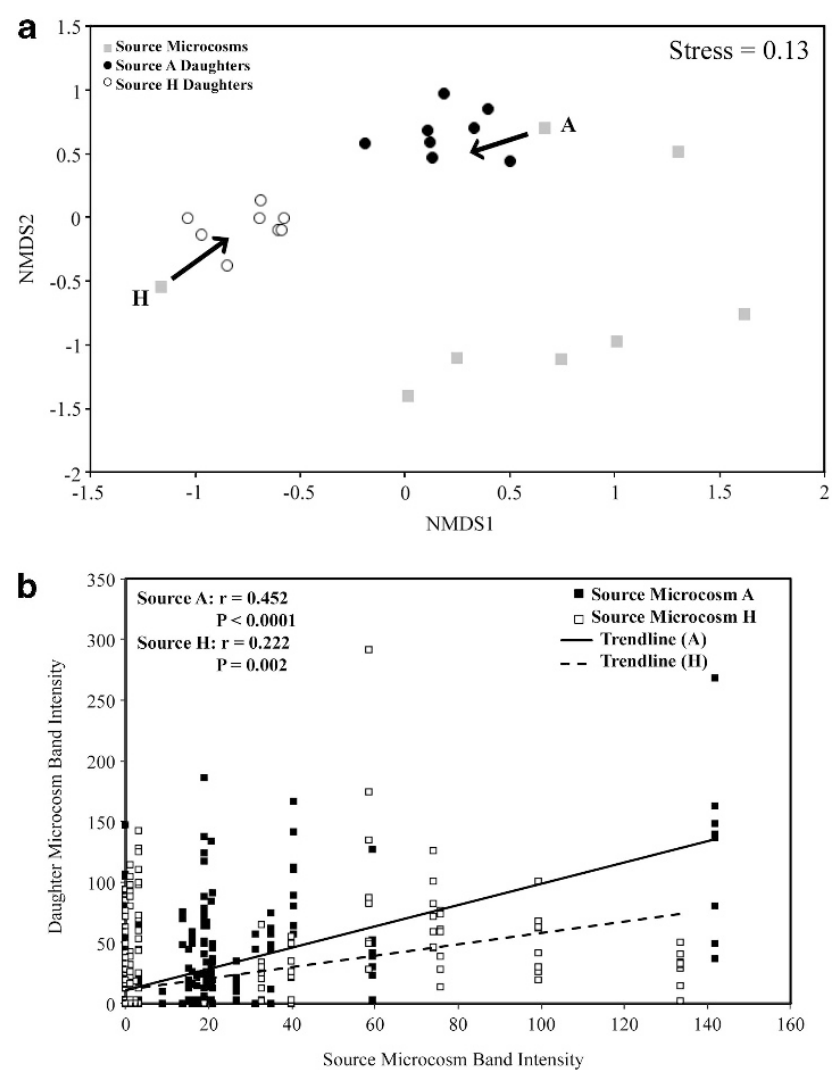

Figure 6 Microcosm community composition is less variable for experiments with pre-conditioned communities. (a) NMDS plot of Bray-Curtis similarity between the DGGE fingerprints of bacterial 16S rRNA gene fragments from eight source microcosm communities $(\mathrm{A}-\mathrm{H})$ and the eight microcosms developed from sources $\mathrm{A}$ and $\mathrm{H}$ (labeled) shows that daughter microcosm communities are more similar to each other and to their source community (arrows) than to a different source or its daughter microcosms. The corresponding fingerprints are shown in Supplementary Figure S6. Analysis of the intensity of individual bands within the bacterial 16S rRNA gene DGGE fingerprints (b) shows a significant correlation between intensity in the source ('parent') microcosm and mean intensity in the replicate 'daughter' microcosms from sources A and $\mathrm{H}(n=8)$.

This suggests an effect of community history stronger than that seen in the previous experiments. Thus, under conditions where the source communities are pre-conditioned to the microcosm environment, community development is more predictable.

An alternative explanation for our results could be that the variability of the final community correlates with the diversity of the starting inoculum, which is lower in our pre-conditioned communities. Random sampling of species from a more diverse (loch) source community might be expected to yield more variable inoculum communities than sampling from a less diverse (microcosm) community. However, although sediment archaeal communities are less diverse than those of Bacteria, we do not observe less variation of Archaea compared with Bacteria in our microcosms. Moreover, we do not observe a significant negative correlation between diversity of the source community and Bray-Curtis similarity of the resultant microcosm communities in our initial set of experiments (Supplementary Figure S7a). To test the hypothesis further, we performed computer simulations of the sampling of inocula from species abundance distributions obtained from pyrosequencing data sets from Loch Leven sediment and from a Blackford Pond microcosm (Supplementary Methods; Supplementary Figure S7b). For inoculum sizes corresponding to those in our experiments (greater than $10^{5}$ individuals), there was little difference in the Bray-Curtis similarity between replicate simulated inocula from loch sediment and from the microcosm. Taken together, these analyses suggest that differential diversity of the source community is unlikely to be the sole explanation for differential microcosm variability in our experiments.

The picture presented in this work, in which colonization of an unfamiliar environment (and hence strong selection) leads to unpredictability, whereas colonization of a familiar environment is more predictable, allows us to rationalize the apparently conflicting results of previous studies on the predictability of microbial community assembly. When an immigrant community is subject to selection criteria very different from those it has previously experienced, as in the colonization of lab microcosms by aquatic microorganisms or colonization of desert habitats by globally-dispersed microbiota, we would expect unpredictable results, and indeed in these cases, divergence of replicate communities is observed (Langenheder et al., 2006; Caruso et al., 2011). Conversely, where the colonizing community has experienced recent selection in a similar environment-as is likely to be the case for parallel bioreactors inoculated with wastewater treatment plant communities and soils transplanted between similar glacier foreland habitatsmore predictable and convergent outcomes would be expected and are indeed observed (Ayarza and Erijman, 2011; Lazzaro et al., 2011).

Importantly, our results show variability between replicate communities at the functional level (assessed in this study by redox potential) as well as at the taxon level. In our microcosms, as in environmental microbial communities (Trosvik et al., 2010; Burke et al., 2011), it is likely that the set of key microbial ecotypes that is present is reproducible across microcosms. However, our results suggest that variability at the taxon level can have a substantial effect on community function, even between replicate microcosms. The variation of broad-scale community function seen in our microcosms is greater than that observed in less spatially-heterogeneous and more short-term laboratory microcosm experiments (Langenheder et al., 2006). This may be because our system has strong, non-linear feedback between community and environmental development: the final environment is largely created by the community itself. In this respect, our model system is similar to real-world microbial ecosystems, such as anaerobic bioreactors 
(Park et al., 2010) and the human gut (Palmer et al., 2007) in which the resident microorganisms are largely responsible for the prevailing environmental conditions.

While we have focused here on unpredictable amplification of rare species as a possible explanation for our observations of divergence between replicate communities colonizing unfamiliar environments, several other explanations are possible. The adaptive evolution of microbial species during our colonization experiments may also contribute to microcosm variability, with rare adaptive mutants having a similar role to strongly selected taxa from the rare biosphere; evolutionary history can also influence the diversification of individual species in a simple community (Knope et al., 2012). The phenomenon of random, low-frequency exit from the non-growing state (Buerger et al., 2012) could also contribute to the amplification of different taxa in different replicate microcosms. Finally, the stochastic birth-death dynamics of low-frequency sub-populations (Black and McKane, 2012; Khatri et al., 2012), perhaps amplified by the effects of viral predation, may also influence the reproducibility of community development.

\section{Conclusions}

By allowing microbial communities sampled from different biogeographical sources to proliferate in replicate pristine microcosms, we have tested whether communities assemble in a predictable or unpredictable manner in a novel environment, and how this depends on the source community. When the community colonizes an unfamiliar habitat, replicate experiments produce very different outcomes, in terms of both community composition (for Bacteria, Archaea and SRB) and function (as measured by redox potential), and initially different inocula diverge rather than converge in composition. In contrast, when a pre-conditioned community colonizes a familiar habitat, community structure is more predictable. Our results show that on colonization of the unfamiliar microcosm environment, rare species, whose presence or absence is likely to vary randomly between replicate inocula, are strongly selected. When our communities instead colonize a familiar environment, the amplification of rare species is less prevalent, community reorganization is reduced and the final community composition is more predictable.

Our work sheds new light on the factors controlling the balance between predictability and unpredictability in microbial community development, and suggests new avenues for control of microbial community assembly. A host of biotechnological and biomedical applications, ranging from wastewater treatment to human gut health, depend crucially on our ability to predict and control the assembly of complex microbial communities from small inocula. Our results suggest that the design of engineered microbial communities is intrinsically limited by the effects of chance, but that preconditioning may prove a useful strategy for minimizing the consequences of this unpredictability.

\section{Conflict of Interest}

The authors declare no conflict of interest.

\section{Acknowledgements}

The authors thank Richard Blythe, Tom Curtis, Neil Gray, Bhavin Khatri, Jana Schwarz-Linek, Wilson Poon, Jim Prosser and Bartlomiej Waclaw for valuable discussions, Kate Heal, Bhavin Khatri and Alex Jackson for assistance with sample collection, Martin Simmen for assistance with NMDS and statistical analysis, Jan Krowkowski for environmental data and Stephen Bridgett and Chris Quince for assistance with the high-throughput sequence analysis. BMS was supported through WP 6 of the EU FP7 WISER project. MEC holds a Royal Society Research Professorship, while RJA was supported by the Royal Society of Edinburgh and by a Royal Society University Research Fellowship. AF was supported by a Darwin Trust Fellowship, and thanks the late Prof. Kenneth Murray for additional support. This work was funded by the Leverhulme Trust under grant F/00158/BX, by the EPSRC under grant EP/E030173, by a NERC CEH small grant and by a Royal Society Research Grant.

\section{References}

Anderson MJ, Gorely RN, Clarke KR. (2008). PERMANOVA + PRIMER: Guide to Software and Statistical Methods. Primer-E: Plymouth, UK.

Ayarza JM, Erijman L. (2011). Balance of neutral and deterministic components in the dynamics of activated sludge floc assembly. Microb Ecol 61: 486-495.

Baptista J de C, Davenport RJ, Donnelly T, Curtis TP. (2008). The microbial diversity of laboratory-scale wetlands appears to be randomly assembled. Water Res 42: 3182-3190.

Becks L, Hilker FM, Malchow $\mathrm{H}$, Jürgens $\mathrm{K}$, Arndt $\mathrm{H}$. (2005). Experimental demonstration of chaos in a microbial food web. Nature 435: 1226-1229.

Benincà E, Huisman J, Heerkloss R, Jöhnk KD, Branco P, Van Nes EH et al. (2008). Chaos in a long-term experiment with a plankton community. Nature 451: 822-825.

Black AJ, McKane AJ. (2012). Stochastic formulation of ecological models and their applications. Trends Ecol Evol 27: 337-345.

Buerger S, Spoering A, Gavrish E, Leslin C, Ling L, Epstein SS. (2012). Microbial scout hypothesis, stochastic exit from dormancy, and the nature of slow growers. Appl Environ Microbiol 78: 3221-3228.

Burke C, Steinberg P, Rusch D, Kjelleberg S, Thomas T. (2011). Bacterial community assembly based on functional genes rather than species. Proc Natl Acad Sci USA 108: 14288-14293. 
Caruso T, Chan Y, Lacap DC, Lau MCY, McKay CP, Pointing SB. (2011). Stochastic and deterministic processes interact in the assembly of desert microbial communities on a global scale. ISME $J \mathbf{5}$ : 1406-1413.

Chase JM. (2003). Community assembly: when should history matter? Oecologia 136: 489-498.

Chase JM. (2007). Drought mediates the importance of stochastic community assembly. Proc Natl Acad Sci USA 104: 17430-17434.

Clarke KR, Warwick RM. (2001). Change in Marine Communities: An Approach to Statistical Analysis, 2nd edn Primer-E: Plymouth, UK.

Donohue I, Jackson AL, Pusch MT, Irvine K. (2009). Nutrient enrichment homogenizes lake benthic assemblages at local and regional scales. Ecology 90: 3470-3477.

Fernández A, Huang S, Seston S, Xing J, Hickey R, Criddle C et al. (1999). How stable is stable? Function versus community composition. Appl Environ Microbiol 65: 3697-3704.

Fuhrman JA, Hewson I, Schwalbach MS, Steele JA, Brown MV, Naeem S. (2006). Annually reoccurring bacterial communities are predictable from ocean conditions. Proc Natl Acad Sci USA 103: 13104-13109.

Geets J, Borremans B, Diels L, Springael D, Vangronsveld J, van der Lelie D et al. (2006). DsrB gene-based DGGE for community and diversity surveys of sulfatereducing bacteria. J Microbiol Methods 66: 194-205.

Graham DW, Knapp CW, Van Vleck ES, Bloor K, Lane TB, Graham CE. (2007). Experimental demonstration of chaotic instability in biological nitrification. ISME J1: 385-393.

Hekstra DR, Leibler S. (2012). Contingency and statistical laws in replicate microbial closed ecosystems. Cell 149: 1164-1173.

Helmus MR, Keller W, Paterson MJ, Yan ND, Cannon CH, Rusak JA. (2010). Communities contain closely related species during ecosystem disturbance. Ecol Letts 13: 162-174.

Jessup CM, Kassen R, Forde SE, Kerr B, Buckling A, Rainey PB et al. (2004). Big questions, small worlds: microbial model systems in ecology. Trends Ecol Evol 19: 189-197.

Jiang L, Patel SN. (2008). Community assembly in the presence of disturbance: A microcosm experiment. Ecology 89: 1931-1940.

Khatri BS, Free A, Allen RJ. (2012). Oscillating microbial dynamics driven by small populations, limited nutrient supply and high death rates. J Theor Biol 314: 120-129.

Knope ML, Forde SE, Fukami T. (2012). Evolutionary history, immigration history, and the extent of diversification in community assembly. Front Microbiol 2: article 273.

Kruskal JB. (1964). Multidimensional-scaling by optimizing goodness of fit to a nonmetric hypothesis. Psychometrika 29: 1-27.

Kuczynski J, Liu Z, Lozupone C, McDonald D, Fierer N, Knight R. (2010). Microbial community resemblance methods differ in their ability to detect biologically relevant patterns. Nat Methods 7: 813-819.

Kurtz JC, Devereu R, Barkay T, Jonas RB. (1998). Evaluation of sediment slurry microcosms for modeling microbial communities in estuarine sediments. Environ Toxicol Chem 17: 1274-1281.
Langenheder S, Székely AJ. (2011). Species sorting and neutral processes are both important during the initial assembly of bacterial communities. ISME $J \mathbf{5}$ : 1086-1094.

Langenheder S, Lindstrom ES, Tranvik LJ. (2006). Structure and function of bacterial communities emerging from different sources under identical conditions. Appl Environ Microbiol 72: 212-220.

Lazzaro A, Gauer A, Zeyer J. (2011). Field-scale transplantation experiment to investigate structures of soil bacterial communities at pioneering sites. Appl Environ Microbiol 77: 8241-8248.

Lepori F, Malmqvist B. (2009). Deterministic control on community assembly peaks at intermediate levels of disturbance. Oikos 118: 471-479.

Madigan RT, Martinko J, Stahl DA, Clark DP. (2011). Brock Biology of Microorganisms, 13th edn. Pearson Prentice Hall: London.

Martiny JBH, Bohannan BJM, Borwn JH, Colwell RK, Fuhrman JA, Green JL et al. (2006). Microbial biogeography: putting microorganisms on the map. Nat Rev Microbiol 4: 102-112.

McCaig AE, Glover LA, Prosser JI. (2001). Numerical analysis of grassland bacterial community structure under different land management regimens by using $16 \mathrm{~S}$ ribosomal DNA sequence data and denaturing gradient gel electrophoresis banding patterns. Appl Environ Microbiol 67: 4554-4559.

Muyzer G, de Waal EC, Uitterlinden AG. (1993). Profiling of complex microbial populations by denaturing gradient gel electrophoresis analysis of polymerase chain reaction-amplified genes coding for 16S ribosomal RNA. Appl Environ Microbiol 59: 695-700.

Myers JA, Harms KE. (2011). Seed arrival and ecological filters interact to assemble high-diversity plant communities. Ecology 92: 676-686.

Nicol GW, Tscherko D, Embley TM, Prosser JI. (2005). Primary succession of soil Crenarchaeota across a receding glacier foreland. Environ Microbiol 7: $337-347$.

Ofiteru ID, Lunn M, Curtis TP, Wells GF, Criddle CS, Francis CA et al. (2010). Combined niche and neutral effects in a microbial wastewater treatment community. Proc Natl Acad Sci USA 107: 15345-15350.

Padua RA, Parrado A, Larghero J, Chomienne C. (1999). UV and clean air result in contamination-free PCR. Leukemia 13: 1898-1899.

Palmer C, Bik EM, DiGiulio DB, Relman DA, Brown PO. (2007). Development of the human infant intestinal microbiota. PLoS Biol 5: 1556-1573.

Park H, Rosenthal A, Jezek R, Ramalingam K, Fillos J, Chandran K. (2010). Impact of inocula and growth mode on the molecular microbial ecology of anaerobic ammonia oxidation (anammox) bioreactor communities. Water Res 44: 5005-5013.

Pedrós-Alió C. (2012). The rare bacterial biosphere. Ann Rev Marine Sci 4: 449-466.

Prosser JI. (2012). Ecosystem processes and interactions in a morass of diversity. FEMS Microbiol Ecol 81: $507-519$.

Prosser JI, Bohannan BJM, Curtis TP, Ellis RJ, Firestone MK, Freckleton RP et al. (2007). The role of ecological theory in microbial ecology. Nat Rev Microbiol 5: 384-392.

Roeselers G, Zippel B, Staal M, van Loosdrecht M, Muyzer G. (2006). On the reproducibility of 
microcosm experiments - different community composition in parallel phototrophic biofilm microcosms. FEMS Microbiol Ecol 58: 169-178.

Sogin ML, Morrison HG, Huber JA, Welch DM, Huse SM, Neal PR et al. (2006). Microbial diversity in the deep sea and the underexplored 'rare biosphere'. Proc Natl Acad Sci USA 103: 12115-12120.

Sokal RR, Rohlf FJ. (1969). Biometry: The Principles and Practice of Statistics in Biological Research. W.H. Freeman and Company: San Francisco.

Trosvik P, Stenseth NC, Rudi K. (2010). Convergent temporal dynamics of the human infant gut microbiota. ISME J 4: 151-158.

Violle C, Pu ZC, Jiang L. (2010). Experimental demonstration of the importance of competition under disturbance. Proc Natl Acad Sci USA 107: 12925-12929.

Weatherby AJ, Warren PH, Law R. (1998). Coexistence and collapse: an experimental investigation of the persistent communities of a protist species pool. $J$ Animal Ecol 67: 554-566.

Wertz S, Degrange V, Prosser JI, Poly F, Commeaux C, Guillaumaud N et al. (2007). Decline of soil microbial diversity does not influence the resistance and resilience of key soil microbial functional groups following a model disturbance. Environ Microbiol 9: 2211-2219.

Whitman WB, Coleman DC, Wiebe WJ. (1998). Prokaryotes: the unseen majority. Proc Natl Acad Sci USA 95: 6578-6583.

Supplementary Information accompanies this paper on The ISME Journal website (http://www.nature.com/ismej) 